\title{
Effect of humidity on liquid-crystalline myelin figure growth using digital holographic microscopy
}

\author{
Rana Mosaviani ${ }^{\mathrm{a}}$, Ali-Reza Moradi ${ }^{\mathrm{a}, \mathrm{b}, \mathrm{c}, *}$, Lobat Tayebi ${ }^{\mathrm{d}, \mathrm{e}, \mathrm{w}^{*}}$ \\ ${ }^{a}$ Department of Physics, University of Zanjan, Zanjan, Iran \\ b Optics Research Center, Institute for Advanced Studies in Basic Sciences, Zanjan, Iran \\ ${ }^{\mathrm{c}}$ Department of Physics, Bilkent University, Cankaya, Ankara 06800, Turkey \\ ${ }^{\mathrm{d}}$ Department of Developmental Sciences, Marquette University School of Dentistry, Milwaukee, WI 53233, USA \\ e Department of Engineering Science, University of Oxford, Oxford OX1 3PJ, UK
}

\section{A R T I C L E I N F O}

\section{Article history:}

Received 30 August 2015

Received in revised form

31 January 2016

Accepted 5 March 2016

Available online 7 March 2016

Keywords:

Myelin figure

Hydration gradient

Humidity

Digital holographic microscopy

Liquid crystals

\begin{abstract}
A B S T R A C T
Dynamics of liquid-crystalline Myelin Figures (MFs) is a multifaceted issue depending on various elements, which have not been fully resolved yet. Our experimental results show that degree of humidity is influential on the initial growth and dynamics of MFs - a factor that was not been carefully considered on MF formations. In this paper, we present a systematic experimental study on the effect of humidity on MF dynamics. Quantitative analysis of MF dynamical behavior was performed using digital holographic microscopy (DHM). Our study reveals that humidifying the initial lipid reservoir has reverse effect on the rate of the growth to the extent that complete saturation of the lipid source prevents MF growth. The phenomenon is explained by the role of hydration gradient during MF formation.
\end{abstract}

(c) 2016 Elsevier B.V. All rights reserved.

\section{Introduction}

There are numerous materials in the world with either understood/useful or unclear/strange behaviors [1-6]. Liquid crystals can be found frequently among materials with strange manners. For example, concentrated mesophases of some membraneforming liquid crystals in water deform readily producing multilamellar cylindrical tubules known as myelin figures (MFs), via a close interplay of fluidity and elasticity. The formation of these complex liquid-crystalline structures and their dynamics are indeed fundamental, unresolved questions and challenging biological features for many researchers since their first observation in 1854 [7-13].

Although MFs can be formed in complex biological systems, their formation and organization bear strong similarity to those obtained during hydration of dried synthetic lipids by water. This in vitro model of MF has proved valuable in studying its fundamental features and dynamics.

Although there are some studies about the MF imaging [14,15],

\footnotetext{
* Corresponding author at: Department of Physics, University of Zanjan, Zanjan, Iran.

** Corresponding author at: Department of Developmental Sciences, Marquette University School of Dentistry, Milwaukee, WI 53233, USA.

E-mail addresses: moradika@znu.ac.ir (A.-R. Moradi), lobat.tayebi@marquette.edu (L. Tayebi).
}

its structure [12,16,17], its initial growth rate [18,19] and coiling behavior [20-25], there are not much investigations explaining the reason of MF formation. However, there are some hypotheses in this regard including a report from Sakurai et al. in which the authors suggested that growth of MFs governs by the collective diffusion of surfactant in solution (diffusive growth model) [8]. Structurally, Buchanan et al. at 2000 hypothesized MFs develop from a blistering instability on a planar bilayer and grow in length as the dry surfactant absorbs water (growth-by-swelling mechanism) [26]. Zou et al., in a report at 2006, shows the previous models cannot cover all the facts regarding the growth and dynamics of MFs [27]. This report claims that MFs formation and growth relies on applying a driving stress. This driving stress can be the hydration gradient, which makes the effect of humidity important (such as the investigation of this paper). In this context any factor that influences the facilitation or elimination of the driving stresses will lead to a change on the MF dynamics. In this letter, we have considered the influence of humidity on MF growth and dynamics. Our experiments show that, besides other factors, growth and dynamics of MFs significantly depend on the degree of humidity in the reservoir of material from which the MFs protrude. However, to the best of our knowledge, this subject which is the focus of this paper, has not yet been systematically studied.

We have performed our quantitative analysis by the use of digital holographic microscopy (DHM) technique on lipidic structure. DHM in a transmission mode can be an effective tool for 
quantitative visualization of phase objects such as living organelles [28-31]. By deriving phase information from the interference pattern between light wave passing through the lipid sample and a reference wave, DHM provides three-dimensional (3D) information on the morphology and volume of MFs with high resolution and at time scales from milliseconds to hours. It enables us to focus on different layers of our samples by numerical methods [32]. Hence, in comparison with conventional microscopy techniques, this method results in more precise and reliable information of MF growth.

Our DHM observation in this study indicated that the humidifying the lipid reservoir (lipid cake) during the incubation time prior to its contact with water can make major influence on the dynamics of MFs and they grow with different rate through lipid cakes with different degrees of humidity. The humidifying effect can reach to the extent that complete saturation of the lipid reservoir prevents the MF growth.

\section{Materials and methods}

The lipid studied in this research, 1-Palmitoyl-2-Oleoyl-Snglycero-3-Phosphocholine (POPC), was purchased in powder type from Avanti and chloroform was used as the solvent. Throughout the experiments deionized water was used to initiate formation of MFs. Clean and dry glass slides and cover slip were used to construct the appropriate chambers for the experiments. Droplets with the volume of $1 \mu \mathrm{l}$ of the solution were put on a glass plate. The samples were kept in vacuum overnight for chloroform evaporation, and then were incubated at $25^{\circ} \mathrm{C}$ in a humid

\section{(a)}

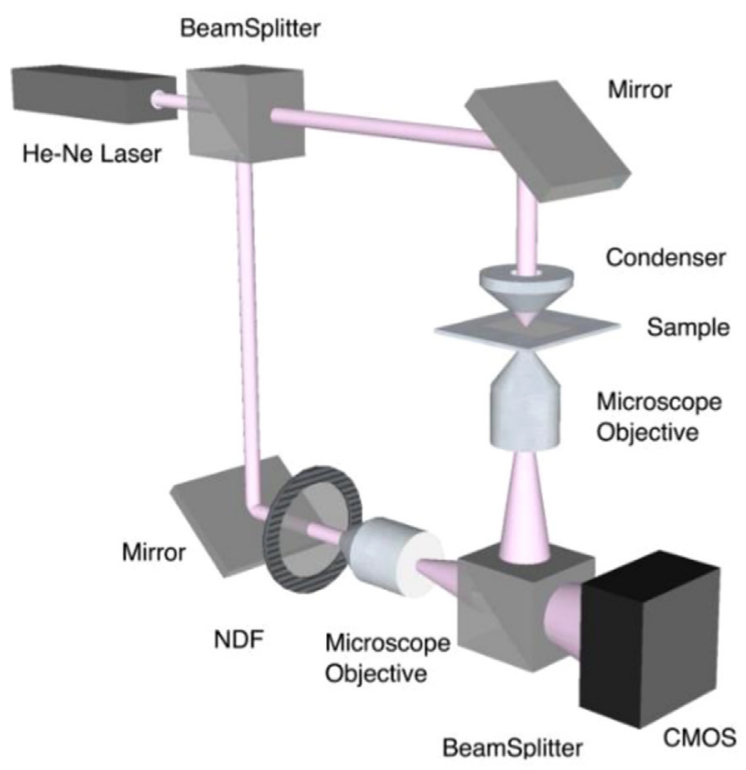

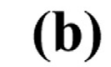

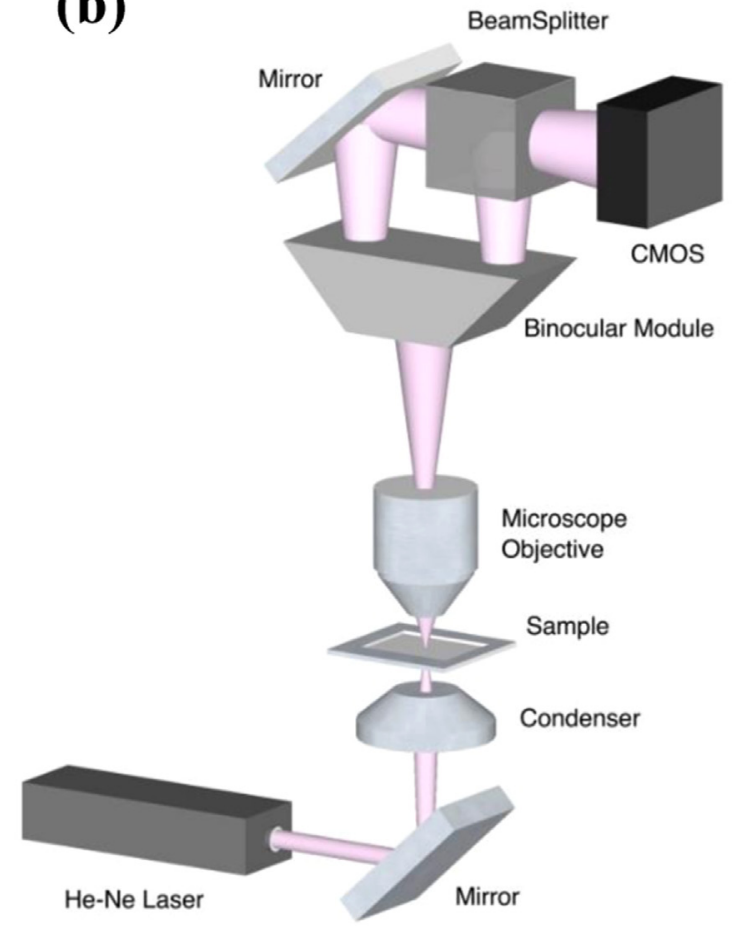

(c)
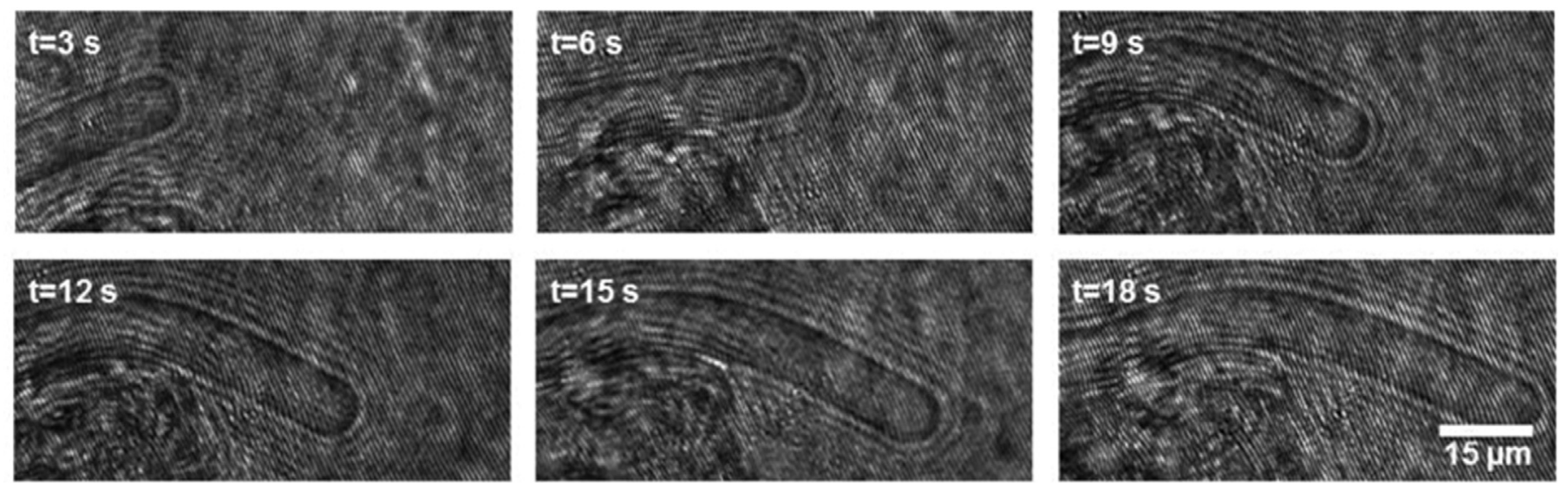

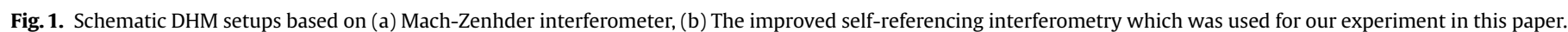

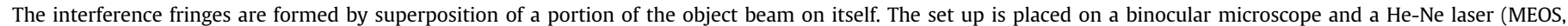

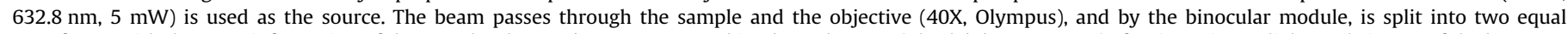

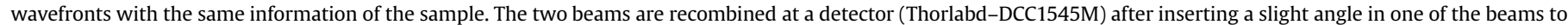
achieve off-axis holograms; (c): Recorded holograms of an MF at $\mathrm{t}=3 \mathrm{~s}$ to $\mathrm{t}=18 \mathrm{~s}$ after its formation starts. 
environment with humidity of $97 \%$ for different time durations from $4 \mathrm{~h}$ to three days to make lipid cakes. MFs were protruded from the lipid cakes after the contact of the cakes with water by the use of a micro-pump at the rate of $10 \mathrm{ml} / \mathrm{h}$. MF growth was observed using a DHM setup in which a laser beam illuminates the object and the magnification is provided by the microscope objective lens and imaging lens combination. A reference beam from the same light source is focused at a point conjugate to the back focal plane of the objective lens, so that it arrives at the detector plane with the same wave front curvature as the object wave. Fig. 1a shows the scheme of a typical Mach-Zehnder interferometer type DHM system for off-axis holography. 3D imaging and investigation of the effect of thermal gradient on MF dynamics have been already performed and reported by the use of such setup in our group [33]. The recorded interference pattern in holography is highly sensitive to environmental and mechanical vibrations. This results in uncorrelated optical path length changes in the two arms of the interferometer, leading to higher noise. However, an improvement can be obtained by eliminating the reference beam toward a self-referencing interferometry [34]. The schematic of such a setup, which has been used in our study for this paper, is shown in Fig. 1b. Since both object and reference beams are derived from the same wave front, the setup becomes much more immune to vibrations. Moreover, less optical components and the possibility to build the system on an up-right conventional microscope provide a low cost and efficient DHM system. The holograms of the MFs were recorded in video format and were converted into image sequences for post processing.

\section{Results and discussion}

Fig. 1c shows recorded holograms of formation and growth of an MF in various time laps. At the beginning of each experiment, in order to remove the background contaminations from the sample container and the optical train, a reference hologram in which no lipid cake is present is recorded.

We utilized the angular spectrum propagation approach in scalar Fresnel-Kirchhoff diffraction theory for numerical reconstruction of the recorded holograms [32]. Within the reconstruction process, the recorded holograms are numerically propagated to the reconstruction plane, from which the phase and intensity of the reconstructed wavefront can be computed. The reconstructed phase pattern is unwrapped by Goldstein's branchcut method to convert it into continuous-phase distribution leading to quantitative phase contrast image of the sample. Fig. $2 \mathrm{a}$ is the $3 \mathrm{D}$ illustration of the associated phase pattern obtained from the reconstructed hologram of a typical MF. Fig. $2 b$ is the projected pattern that can be used to calculate the area covered by MF. Assuming negligible changes for refractive index, the thickness at an arbitrary point on the MF can also be calculated and hence the volume can be obtained. Fig. 2c shows the thickness distribution (a)

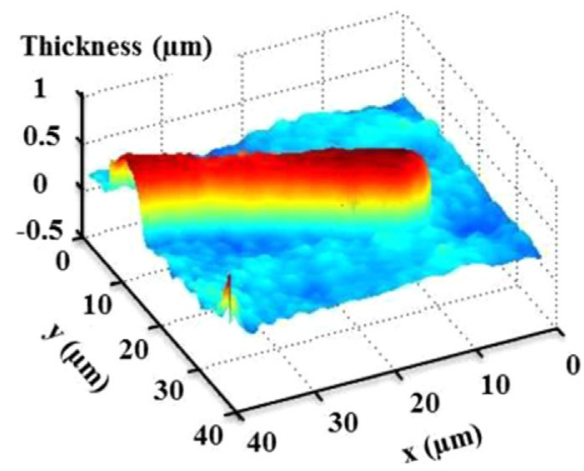

(b)

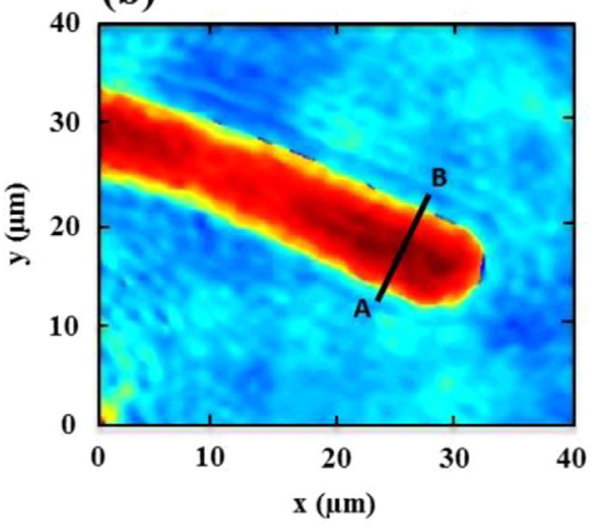

(c)

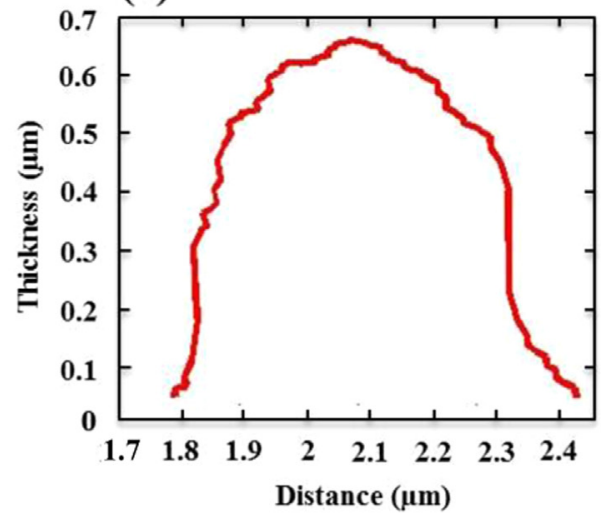

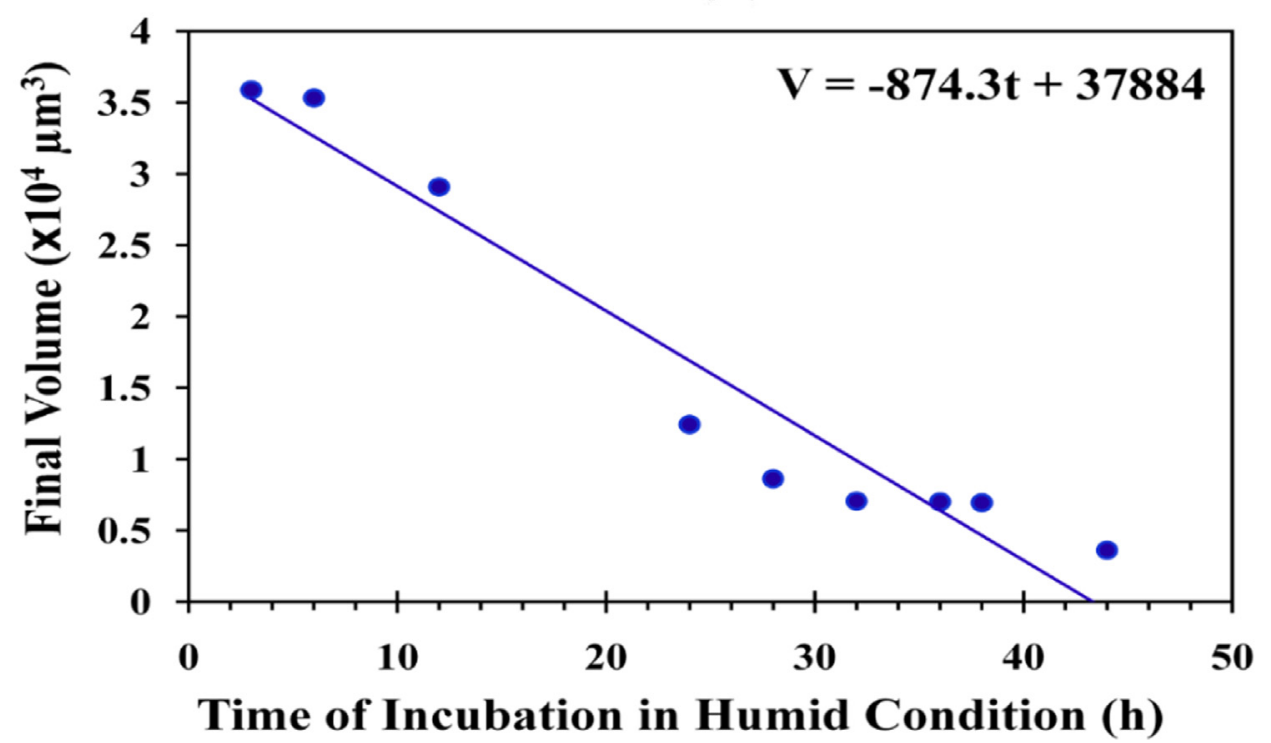

(d)

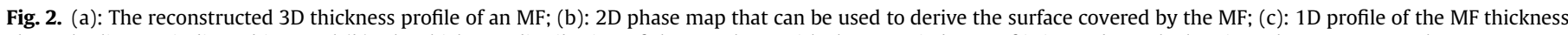

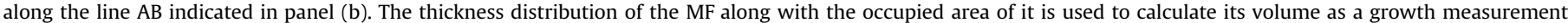
parameter; (d): The final volume of MFs vs. incubation time showing an approximate linear dependence. The slope of the fitted line is equal to $-874.3 \mu \mathrm{m}^{3} \mathrm{~s}^{-1}$. 
along the line specified in Fig. 2b. The reconstruction process was repeated for all holograms that were recorded at the rate of $25 \mathrm{fps}$ for each sample. Thickness, length, surface, and volume of the MFs were obtained from the phase patterns for all the MFs in the imaging field of view, and in each sample the average value of several measurements of all MFs was reported. The evolution of these parameters in time for different experimental conditions can be used to follow the MFs dynamics.

Our investigation indicates that the growth and dynamics of MF changes significantly for different incubation durations in humid condition.

First we measured the final volume of the protruded MFs from lipid cakes with various incubation times, which are plotted in Fig. 2d. Measurements were performed on several samples in at least two-hour time intervals. The volumes are calculated and reported for the samples until they reach the stable values. Our experiments reproducibly indicated that increasing the initial incubation time of lipid cakes $(t)$ dramatically decreases the final volume of MFs (V) protruded from those cakes (Fig. 2d). The final volume of MFs has approximately a linear dependence to the duration of incubation with sharp negative slope. We consistently observed that the $\mathrm{V}$ reached zero after around $43 \mathrm{~h}$ incubation, which means formation of no MF. For the durations longer than $43 \mathrm{~h}$ no evidence of growth was observed. Fig. 3a presents a closer look to the dynamics of MFs protruded from lipid cakes with various incubation time by plotting the growth of the MF lengths. As shown in Fig. 3a, the best fitted curves of the MF length vs. time can indicate that the growth rate of the each MF decreases by increasing the incubation time. Since many scientists believe that MFs grow as $L \propto \sqrt{t}$, we fitted the curves based on that in Fig. $3 \mathrm{~b}$, which shows $40 \mathrm{~h}$ extra incubation time can lead to 9 times decay in the slope of the MF growth. Thus, not only the MF is smaller when protruded from highly humidified cakes but also the rate of its growth is significantly decreased.

Our scale for considering the degree of humidity in lipid cakes is the time of incubation of the initial lipid drops in the humid environment. This incubation may cause the slow penetration of water molecules by lipids stacks leading to the self-assembly of lipid multilayer.

The reason of MF formation is not yet clearly understood, although there are a few theories in this regard. Sakurai et al. suggested that MF growth governs by the collective diffusion of lipid, or in general a surfactant, in solution (diffusive growth model) [8]. Structurally, Buchanan et al. hypothesized that MFs develop from a blistering instability on a planar bilayer [10]. Zou et al. in a report published on 2006 expressed that MFs only form and grow at the presence of a driving stress [27]. The result of our experiments in this paper provide an evidence in the favor of the latter theory in which the gradient of hydration between the source of lipid and the environment can act as a driving stress. When the lipid cake is fully hydrated after elongated period of incubation in humid condition, the hydration gradient can be negligible to be considered as an external driving force for the formation of MFs. In the other word the absence of hydration gradient prevents the MF growth.

\section{Conclusion}

Using digital holographic microscopy technique, we have studied the effect of humidifying the initial lipid source on the formation and growth of MFs. Employing DHM provides detailed information of the 3D profile variations of the MF morphology by time. Our experimental observation indicates that humidifying the

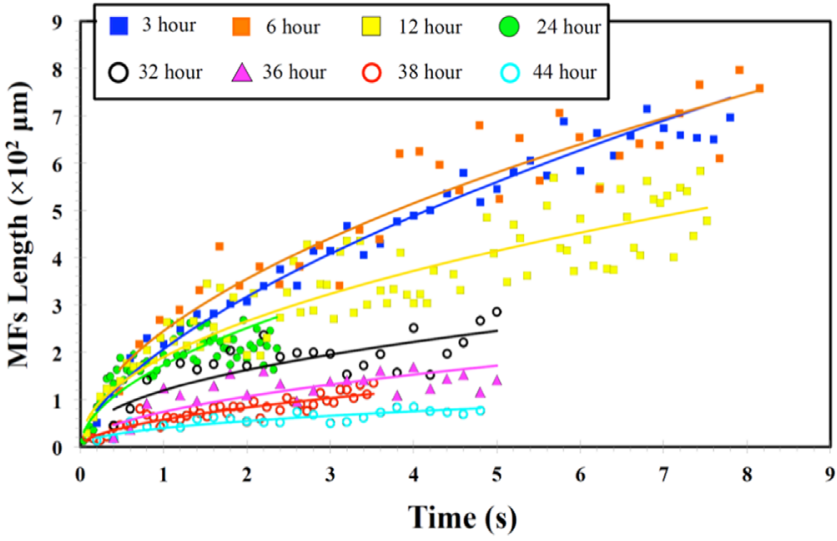

(a)

$$
\begin{aligned}
& L_{3 h}=206.33 t^{0.6204} \\
& L_{6 h}=244.65 t^{0.5364} \\
& L_{12 h}=189.55 t^{0.4858} \\
& L_{24 h}=172.82 t^{0.5374} \\
& L_{32 h}=118.92 t^{0.4484} \\
& L_{36 h}=73.894 t^{0.5227} \\
& L_{38 h}=57.418 t^{0.5303} \\
& L_{44 t h}=39.553 t^{0.4602}
\end{aligned}
$$

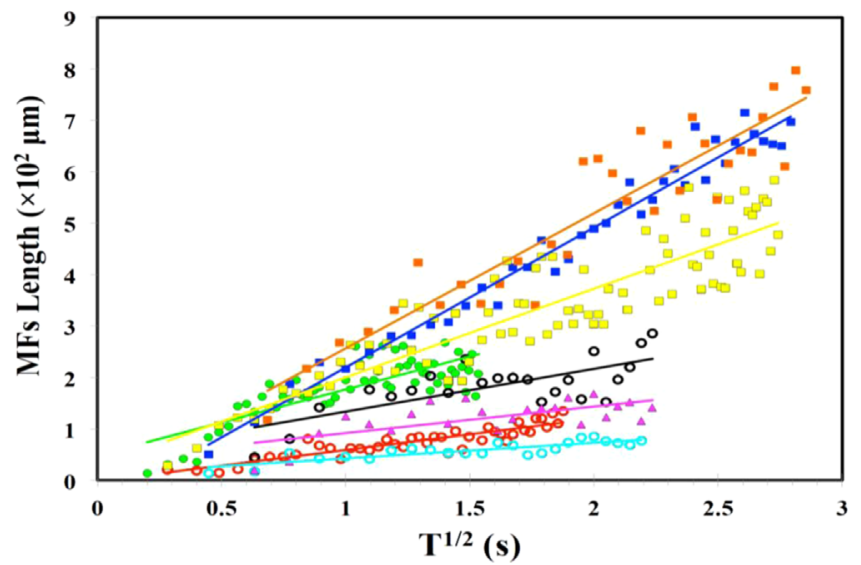

(b)

$$
\begin{aligned}
& \mathrm{L}_{3 \mathrm{~h}}=272.01 \sqrt{ }-52.464 \\
& \mathrm{~L}_{6 \mathrm{~h}}=262.31 \sqrt{ }-5.5129 \\
& \mathrm{~L}_{12 \mathrm{~h}}=172.27 \sqrt{ }+27.988 \\
& \mathrm{~L}_{24 \mathrm{~h}}=128.18 \sqrt{ }+48.543 \\
& \mathrm{~L}_{32 \mathrm{~h}}=83.136 \sqrt{ }+50.526 \\
& \mathrm{~L}_{36 \mathrm{~h}}=51.593 \sqrt{ }+40.262 \\
& \mathrm{~L}_{38 \mathrm{~h}}=60.825 \sqrt{ }-1.8058 \\
& \mathrm{~L}_{44 \mathrm{~h}}=30.353 \sqrt{ }+12.218
\end{aligned}
$$

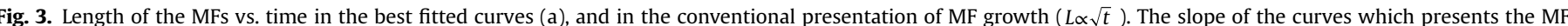
growth rate decreases by increasing the humidity. Our experiments reproducibly indicated that after around $43 \mathrm{~h}$ incubation no MF can protrude from the lipid cake. 
initial lipid reservoir has reverse effect on the rate of the growth. More specifically, $40 \mathrm{~h}$ incubation in 97\% humidity can 9 times decrease the growth rate, and no MF grows from lipid cakes incubated for approximately $43 \mathrm{~h}$ and more. Our finding are in agreement with a theory of MF growth that indicates the necessity of a driving force for MF formation. In our case, the driving force is the hydration gradient.

\section{Acknowledgement}

The authors are thankful of the support from Delta Dental, National Science Foundation (NSF, Grant no.CMMI-1363485) and Marquette University Strategic Innovation Fund.

\section{References}

[1] T. Tomita, K. Kuga, Y. Uwatoko, P. Coleman, S. Nakatsuji, Strange metal without magnetic criticality, Science 349 (2015) 506-509.

[2] R.D. James, Materials science: magnetic alloys break the rules, Nature 521 (2015) 298-299.

[3] S.T. Lagerwall, Orthoconic liquid crystals-a case study, Adv. Colloid Interface Sci. 208 (2014) 1-9.

[4] M. Mozafari, D. Vashaee, L. Tayebi, M. Mehraien, Electroconductive Nanocomposite Scaffolds: A New Strategy Into Tissue Engineering and Regenerative Medicine, INTECH Open Access Publisher, InTech Janeza Trdine 951000 Rijeka, Croatia, European Union, 2012.

[5] L. Tayebi, Y. Ma, D. Vashaee, G. Chen, S.K. Sinha, A.N. Parikh, Long-range Interlayer alignment of Intralayer domains in stacked lipid bilayers, Nat. Mater. 11 (2012) 1074-1080.

[6] E. Salahinejad, M. Hadianfard, D. Macdonald, I. Karimi, D. Vashaee, L. Tayebi, Aqueous Sol-gel synthesis of zirconium titanate $\left(\mathrm{ZrTiO}_{4}\right)$ nanoparticles using chloride precursors, Ceram. Int. 38 (2012) 6145-6149.

[7] R. Virchow, Virchows Arch. Pathol. 6 (1854).

[8] I. Sakurai, Y. Kawamura, Growth mechanism of myelin figures of phosphatidylcholine, Biochim. ET Biophys. Acta (BBA). Biomembr. 777 (1984) 347-351.

[9] L.N. Zou, Myelin figures: the buckling and flow of wet soap, Phys. Rev. E. 79 (2009).

[10] M. Buchanan, J. Arrault, M.E. Cates, Swelling and dissolution of lamellar phases: role of bilayer organization, Langmuir 14 (1998) 7371-7377.

[11] J.R. Huang, L.N. Zou, T.A. Witten, Confined multilamellae prefer cylindrical morphology, Eur. Phys. J. E 18 (2005) 279-285.

[12] L. Tayebi, M. Mozafari, D. Vashaee, A.N. Parikh, Structural configuration of myelin figures using fluorescence microscopy, Int. J. Photoenergy 2012 (2012).

[13] H. Mohit, C. Ashim, C. Manohar, B. Jayesh, Myelin growth and coiling, Colloids Surf. A: Phys. Eng. Asp. 205 (2002).
[14] W. Stoeckenius, An electron microscope study of myelin figures, J. Biophys. Biochem. Cytol. 5 (1959) 491-500.

[15] H. Wang, Y. Fu, P. Zickmund, R. Shi, J.-X. Cheng, Coherent anti-stokes Raman scattering imaging of Axonal myelin in live spinal tissues, Biophys. J. 89 (2005) $581-591$.

[16] K. Peddireddy, P. Kumar, S. Thutupalli, S. Herminghaus, C. Bahr, Myelin structures formed by Thermotropic smectic liquid crystals, Langmuir 29 (2013) 15682-15688.

[17] I. Sakurai, T. Suzuki, S. Sakurai, Cross-sectional view of myelin figures, Biochim. ET Biophys. Acta (BBA)-Biomembr. 985 (1989) 101-105.

[18] H. Dave, M. Surve, C. Manohar, J. Bellare, Myelin growth and initial dynamics, J. Colloid Interface Sci. 264 (2003) 76-81.

[19] K. Mishima, T. Ogihara, M. Tomita, K. Satoh, Growth rate of myelin figures for phosphatidylcholine and phosphatidylethanolamine, Chem. Phys. Lipids 62 (1992) 87-91.

[20] K. Mishima, K. Fukuda, K. Suzuki, Double helix formation of phosphatidylcholine myelin figures, Biochim. ET Biophys. Acta (BBA)-Biomembr. 1108 (1992) 115-118.

[21] M. Haran, A. Chowdhury, C. Manohar, J. Bellare, Myelin growth and coiling, Colloids Surf. A: Phys. Eng. Asp. 205 (2002) 21-30.

[22] V. Frette, I. Tsafrir, M.-A. Guedeau-Boudeville, L. Jullien, D. Kandel, J. Stavans, Coiling of cylindrical membrane stacks with anchored polymers, Phys. Rev. Lett. 83 (1999) 2465.

[23] C. Santangelo, P. Pincus, Coiling instabilities of multilamellar tubes, Phys. Rev. E. 66 (2002) 061501.

[24] J.-R. Huang, Theory of myelin coiling, Eur. Phys. J. E. 19 (2006) 399-412.

[25] K.-C. Lin, R.M. Weis, H.M. McConnell, Induction of helical liposomes by $\mathrm{Ca}^{2+}$ mediated intermembrane binding, Nature (1982).

[26] M. Buchanan, S.U. Egelhaaf, M.E. Cates, Dynamics of interface instabilities in nonionic lamellar phases, Langmuir 16 (2000) 3718-3726.

[27] L.N. Zou, S.R. Nagel, Stability and growth of single myelin figures, Phys. Rev. Lett. (2006) 96

[28] U. Schnars, W.P. Jüptner, Digital recording and numerical reconstruction of holograms, Meas. Sci. Technol. 13 (2002) R85.

[29] M.K. Kim, Principles and techniques of digital holographic microscopy, J. Photonics Energy (2010) 018005-50.

[30] I. Moon, M. Daneshpanah, A. Anand, B. Javidi, Cell identification computational 3-D holographic microscopy, Opt. Photonics News 22 (2011) 18-23.

[31] P. Marquet, B. Rappaz, P.J. Magistretti, E. Cuche, Y. Emery, T. Colomb, et al., Digital holographic microscopy: a noninvasive contrast imaging technique allowing quantitative visualization of living cells with subwavelength axial accuracy, Opt. Lett. 30 (2005) 468-470.

[32] A. Anand, V.K. Chhaniwal, B. Javidi, Real-time digital holographic microscopy for phase contrast 3D imaging of dynamic phenomena, J. Disp. Technol. 6 (2010) 500-505.

[33] N. Fathi, A.-R. Moradi, M. Habibi, D. Vashaee, L. Tayebi, Digital holographic microscopy of the myelin figure structural dynamics and the effect of thermal gradient, Biomed. Opt. Express 4 (2013) 950-957.

[34] S. Ebrahimi, A.-R. Moradi, A. Anand, B. Javidi, T. Elhanan, D. Yelin, et al., Digital holographic microscopy with Coupled optical fiber trap for cell measurement and manipulation, Opt. Lett. 39 (2014) 2916-2919. 